\title{
The Professional Contribution of Chiropractors to Danish elite football clubs: A qualitative exploration of role and perceived value in an interprofessional service provision context.
}

Joachim Hostrup

University of Southern Denmark

Anders Koza

University of Southern Denmark

Corrie Myburgh ( $\nabla$ cmyburgh@health.sdu.dk)

University of Southern Denmark https://orcid.org/0000-0002-7741-1313

\section{Research}

Keywords: Sports chiropractic, Sports medicine, interprofessional practice, Healthcare team

Posted Date: May 22nd, 2020

DOl: https://doi.org/10.21203/rs.3.rs-29241/v1

License: @ (1) This work is licensed under a Creative Commons Attribution 4.0 International License.

Read Full License 


\section{Abstract}

Background: Interprofessional team-based care has been widely adopted in elite level athletic health and performance practice. And although chiropractors can claim some penetration as health care service providers in high level sport, their position as valued members of interprofessional health care teams, especially those built around traditional medical organisational structures, is unclear.

In Denmark, the mainstream provider status of chiropractors is more favourable than in most parts of the world. Moreover, chiropractors have been identified as service providers in the Danish premiere football league (Super Liga). However, little is currently known about the professional contribution made by chiropractors in this powerful sporting context.

As a first step to map the professional practice landscape of Danish sports chiropractors, this investigation sought to explore the perceived role and perceived value of chiropractors as service providers in elite Danish football clubs.

Methods: A comparative qualitative case study using semi-structured individual interviews was conducted. Six Danish Superliga clubs were purposively sampled to compare and contrast instances where chiropractors were absent from the health care team, contracted as in-house health care team members or engaged as external service providers. Triangulated responses were solicited from healthcare co-ordinators, chiropractors and athletes within each club's organisation through short semi-structured individual interviews. The audio-recorded responses were transcribed verbatim and thematically analysed using a framework approach.

Results: Inductive and deductive coding revealed 14 unique codes, which were further organised into 4 code families relating to the role of chiropractors, benefits of utilizing chiropractic care and facilitators and barriers to interprofessional practice. From this framework 3 themes were abstracted, these being: "Broadening horizons", "In-house preferred to take-away" and "Already covered, or even necessary?".

Conclusion: In this practice context, chiropractors fill the role of musculoskeletal health care service providers. Their perceived value stems from additional expert disciplinary knowledge, improved diagnostic triage and increased treatment flexibility. However, where not utilized, the role of the spinal health expert is questioned and when acknowledged, limited to that of a technician/therapist. It is unclear whether chiropractors can claim core team member status. Further exploration of this interesting context of IPP practice is warranted.

\section{Background}

Interprofessional health care practice (IPP) occurs when practitioners from different backgrounds combine their unique disciplinary knowledge, in an effort to provide the highest quality of service to people, living with a particular health care challenge $(1,2)$. IPP has gained wide support as an innovative 
approach to coping with future health care delivery demands and significant efforts have been made to both increase and optimise its occurrence (3-5).

In high stakes elite and professional sport, organizations gamble on identifying potential ' $X$ factor' athletes capable of providing a competitive edge, ultimately translating into the realization of performance goals and/or profitability (6). Sidelined athletes give a poor return on investment, and therefore in instances of athletic injury, rapid return-to-play is prioritised (7). With this strategic goal in mind, sporting organisations with the financial means, now commonly draw expertise from different professions into inter-professional health care teams (IPHCT) (8). The perpetual drive for discovering competitiveness edges appears to extend into health service delivery. Consequently, non-traditional occupational groups with perceived value, have an opportunity to find inclusion in the IPHCT. This context therefore extends IPP beyond that of ordinary (mainstream) health care providers to include professions classified as so-called complementary and alternative medicine (CAM) provider groups.

In this regard chiropractic represents an interesting exemplar as chiropractors, in most contexts, are classified as CAM service providers and furthermore and claim a role as service providers in elite level athletic health and performance $(9,10)$. Generally, this success is attributed to the need expressed by athletes for chiropractic services, and in formal competition settings, the acceptance of a subordinate and limited role as manual therapist (11).

Seen from an IPP perspective, chiropractors appear to have gained access to elite level sporting events, not due to a clear role and perceived value as part of the IPHCT, but rather as beneficiaries of an athletecentered policy of service provision, powerful enough to override traditional sports medicine organisational structures, which tend to view chiropractors as redundant (11-13). This situation is suboptimal for the development of IPP as the additional health outcomes benefit originates from a culture where the contributions of individual members are equally valued (4).

In Denmark, chiropractic has transitioned into a mainstream service provider with higher levels of mainstream integration being observed (14). It is therefore conceivable that this general state of increased inclusion has manifested in the elite athletic health and performance practice. This context of practice has, however, not been explored.

Football is Denmark's most popular (362.418 active football players in 2018) (15) and wealthiest sport (Denmark's top league clubs currently carry a combined value of just over 186 million euros (16). Moreover a recent audit of medical staff indicated that seven out of 17 teams $(41,2 \%)$ associated with the Superliga, formally engage the service of a chiropractor (17). Given the economic capacity to employ an extended IPHCT and the presence of chiropractors in this context of care, an intriguing question arises: 'Are chiropractor perceived as novelty practitioners, or is there evidence that the profession has begun to find traction as a member of the IPHCT with a defined role and perceived value?'

With the above in mind, the aim of this investigation was to explore the role and perceived value of chiropractors to Danish elite football clubs. And additionally, to explore barriers and facilitators to IPP 
involving chiropractors.

\section{Methods}

Study design

A comparative qualitative case study was conceptualized to explore a particular case of health care service provision (18).

\section{Theoretical stance}

This design was underpinned by a constructivist stance, as we sought to understand the roles and consequent value of chiropractors by creating a meaningful co-construction of the experiences of participant stakeholders (19).

\section{Participants}

In order to adequately observe this complex social action (the unit of analysis) adequately, we triangulated data from three units of observation; these being health care co-ordinators, chiropractors and athletes (20). The study was bracketed to these data sources in order to focus feedback on insights relating to IPP, but also role and perceived value (21).

\section{Sampling}

We used a maximum variation strategy to identify from the Danish Superliga organisation, clubs that both included and excluded chiropractors as part of their healthcare team (22). To achieve this, club websites were visited in order to retrieve healthcare co-ordinators or club administration contact information. A request for participation was then e-mailed to the co-ordinators. In the event that a positive response was received, the health care co-ordinators mediated access and eventual recruitment of athletes and chiropractors (where appropriate) (23).

Predicting a sample size a priori is not an exact process as the eventual sample depends on whether enough of rich and thick data, has been procured so as to make further sampling meaningless (24). To address this issue, we sought to include at least two instances, where chiropractors were utilized and two where they were not. It was therefore expected that a sample of approximately 10 respondents would be required. 


\section{Data collection}

Participant perceptions and experiences were captured through individual interviews appropriate for making meaning of relevant social and personal life experiences (25). Given the geographical spread of clubs, both face-to-face and telephonic interviews were conducted, to ensure sampling flexibility $(26,27)$. Interviews were of the semi-structured variety, suitable for when only interview data is collected (28). Interview guides were constructed, consisting of open-ended questions designed to elicit responses around roles (functions) and values (benefits/impact) in general and more specifically barriers and facilitators to IPP. All questions were supported by probes, to encourage further elaboration relevant to the responder groups.

Interviews were audio-recorded, transcribed verbatim into word text documents and anonamised. In this regard health care co-ordinators, athletes and chiropractors were assigned the acronyms 'HC', 'AT' and ' $\mathrm{CH}$ ', respectively. Key quote position in transcript was indicated with line numbers, designated with the prefix 'L'. And all key quotes were contextually translated into English.

\section{Data analysis}

Data were analyzed using thematic framework analysis approach (29). From the initial interviews, two authors, JH and AK independently created a code book by inductively coding meaningful segments of text. By means of code definition comparison and a consensus process mediated by the third author, CM, a common code book was negotiated. In accordance with the constant comparison method (30), as new codes emerged in subsequent interviews, either due to new observations or as a result of more abstract (axial) coding, the code book was amended and previous interviews scanned for new code presence. Codes were subsequently further organised into code families, and key messages abstracted to reveal the eventual key themes.

Finally, member check was performed, by asking four participants to affirm that the findings as reflective of the context of practice (31).

\section{Ethics}

Participants were provided with an information package before an interview was arranged and informed consent was sought from all participants before interviewing commenced. Moreover, the study followed 
the guidelines of the Danish Data Protection Agency and was conducted with the approval of the science and ethics committee of the Danish region of Southern Denmark (Reference number: 20202000-85).

\section{Results}

\section{Results of the sampling}

Ten individual interviews were conducted between $13^{\text {th }}$ of September 2019 and $8^{\text {th }}$ of November 2019, lasting on average 17 minutes per interview. Six were conducted at club houses, two at chiropractic clinics, one at a public café and one via telephone. As can be seen from figure 1, below, the sampling process identified instances with and without a chiropractor. However, a third category resulted as clubs employed chiropractors as in-house staff members and external health care providers. An "in-house therapists" was defined as having fixed weekly sessions at the site of the club, where as to the "external service provider" functioned on an ad hoc basis. One health care co-ordinator (HC4) agreed to be interviewed, but became unavailable for a face-to-face or telephonic interview during the data collection period. Interviews with chiropractors and athletes were conducted after interviews of the healthcare coordinator of the same clubs had been held.

Figure 1: Flowchart of the sampling process

\section{Thematic framework}

Based on participant responses, 14 individual codes were identified and organised into four code families, which included barriers and facilitators. From the codified data, 3 themes were abstracted, these being 'broadening horizons', 'in-house preferred to take-away' and 'already covered or even necessary?'. The organisation of the thematic framework is illustrated in figure 2, below.

Figure 2: Organisation of data indicating emergence of themes.

\section{Broadening horizons}

Generally speaking, health care co-ordinators engaged the chiropractor's services with aim of bolstering the expertise level around spine-related problems, but also to gain a different/new approach to assessment and treatment. According to $\mathrm{HC} 1$ and 5 :

I found that he would be able to complement me and the other physiotherapists. Particularly with the knowledge of the back and neck. I felt we needed to improve on that subject (HC1; L52-L54).

and 
They of course have some special competence regarding manipulation. They have special skills in assessment of, for example, back patients regarding how a back moves, how an SI [sacro-iliac] joint moves and how you assess that... But they just have some other techniques and other approaches, that make it possible to cover the issue in the best way possible (HC5; L44-49).

A different approach was thought to bring other aspects to the pooled knowledge, enabling the IPHCT to make more informed decisions. According to $\mathrm{HC} 1,2$ and 5:

The feedback from the players has been really good but also in the team, where we complement each other well, and something extra has been brought (HC1; L61-62)

Otherwise I would say the overall effect I have felt is that we have gotten an extra pair of hands and it is in regard to sparring and in multidisciplinary teamwork that they have contributed the most to me ( $\mathrm{HC2}$; L107-109).

and

I think there was a need for their knowledge and what they could do (HC5; L122).

Added value over previous service provision model was thought to lie in covering a broader spectrum of healthcare, resulting in a greater likelihood of pin pointing 'the right diagnosis' (HC5; L47-49). This discourse is highlighted by $\mathrm{HC} 2$ and 3 , respectively:

We cover everything, so nothing is left uncertain, because they [chiropractors] have a different sense and a different way of approaching the joints than I do. And it complements each other well, I think. I think we are covering a broader spectrum, also in treatment and I think that is where the gain is (HC2; L114-117).

and

I think it is important to cover a broad spectrum of what you do. We have two physios and a massage therapist now, ... and then we send people externally, if we are missing something specific (HC3; L46-47).

Health care co-ordinators engaged chiropractors as spine-related musculoskeletal health experts offering specific discipline-specific knowledge regarding biomechanical injury mechanisms, patient assessment and diagnosis and competencies in conservative manual interventions such as manipulation, mobilization, dry needling. Their rationale for adding the chiropractor is to broaden the shared pool of knowledge with the expressed benefit of better diagnoses and more comprehensive management.

\section{In-house preferred to take-away}

Our data indicates that clubs initially made use of the chiropractic service on an ad hoc basis. However, once established, the availability of the chiropractor became an issue for IPP, in particular effective care 
co-ordination. In this regard, consensus existed that contracting chiropractors as internal service provider, was a distinct improvement compared to earlier ad hoc utilization. This service provision evolution is illustrated in the following discourse from club 2:

... as time went by, I tried to see if we could improve, of course for the football club, but the chiropractors also wanted to contribute more. And then they started to come to the site of the club as well... it has evolved because that worked so well for us. We could make it, so the players were closer to treatment, ..., that made it easier for the players and actually for the chiropractors as well, to keep control of things. Instead of seeing the players sometimes, and then not for 2-3 weeks (HC2; L39-47).

The interdisciplinary teamwork is good, especially in the later years where we have attended the training camps, ..., that just makes us know each other's strengths and weaknesses much better. ... And it works with equality with respect for each other (CH2; L77-81),

I know that they will come tomorrow, so there he can crack my back and loosen my hip, then I maybe get the massage therapist to do that and that, so that I do not get double treatment... Planning wise it is also easy for us ... That is nice" (AT2; L32-37).

From a service delivery perspective, this developing relationship was perceived as beneficial to athletes as service delivery could more readily include curative and preventative care. In this regard HC 1 stated:

... the chiropractor also sees the same people repeatedly and not only when they get injured. Earlier it was only when they got injured. Let's say they had an acute lumbago, then they would be sent to a chiropractor. Now he is more implemented every day in everything (HC1; L29-36).

A pragmatic benefit of having chiropractors as internal therapists was 'getting extra pairs of hands', so that there is 'more time for immersion' with each athlete (HC2; L102-104). This view was echoed by HC5, who saw shifting to in-house chiropractic services as a wish list item, stating: "We could use some extra hands and some extra time for immersion" (HC5; L137-138).

For the chiropractor, attaining in-house service provider status provided a clear benefit of direct access to athletes. These benefits included athletes developing a better understanding of 'what we [chiropractors] are good at and what the physio is good at ' $\mathrm{CH} 2 ; \mathrm{L} 50-53)$. Moreover, care could be initiated timeously. In this regard $\mathrm{CH} 1$ observed:

... the previous physios were not fans of chiropractors, it was the players who needed to ask to be seen by a chiropractor ... That was a problem because then we see the patient too late ( $\mathrm{CH} 1 ; \mathrm{L} 41-44)$.

Shifting chiropractors from external to in-house practitioners, provided health care co-ordinators with care continuity benefits. Moreover, having more practitioners available provided an additional advantage to individualized care strategies. And direct access to athletes provided the chiropractor with a better opportunity to timeously initiate appropriate care. 
Already covered, or even necessary?

In the two instances of clubs not utilizing chiropractors, services deemed relevant to a chiropractors were instead assigned to an osteopath. In this regard HC6 and 7 stated:

... we have had osteopaths all along in the club instead of chiropractors... which you could argue on some points are similar (HC6; L6-10).

and

We do not have a chiropractor employed, but we have an osteopath employed and a part of the osteopath education is also chiropractic - at least in some ways with manipulations etc... (HC7; L2-3).

For HC7, the osteopath 'only does manual therapy when he is with us' (HC7; L32-33) and mainly with a preventative focus. In this regard $\mathrm{HC} 6$ and $\mathrm{HC} 7$ elucidated this role, stating:

... he [the osteopath] has no part in rehabilitation. The role he has is that some of the long-term injured players - some of them who are maybe back in training, but still have some irritations - I send them to him (HC7; L32-37).

The osteopath covers the preventive correction. What I see as the strength of the osteopath is to look at alignments, the whole person and where there are things that are ..., badly compensated ..., that we maybe can try to correct and see if it is compensated better before you start with for example strength training and all sorts of other things (HC6; L53-57).

Interestingly both respondents, despite their perceptions of chiropractors and osteopaths overlapping significantly, admitted to having a very limited knowledge of the chiropractors' scope of practice. Specifically, HC6 and 7 stated:

But I will say, I know nothing about chiropractic (HC6; L72)

and

I do not know very much of the chiropractic education... I do not know the chiropractor's role in rehabilitation and how good they are at that - and that is probably why it is physiotherapists we employ (HC7; L54-58).

From a practitioner utilisation and cost-effectiveness perspective, $\mathrm{HC} 7$ argued that smaller clubs are better off with a lean health care team, rather than attempting to offer extended services. In this regard HC 7 argued that:

This is a club with not that much money... There is [only]money for two full time employees, and I prefer that instead of me being full time and four others sharing the other days, ... And from those criteria I think 
it makes more sense to have two full time physiotherapists where one of them is an osteopath, than having a chiropractor employed (HC7; L14-19)

Finally, the seriousness of spinal problems as factors affecting training and performance in football were questioned and as a result the need for a practitioner with a limited spinal focus. For HC 7: “... we do not experience players who have an issue with their low back being out of training. That is very rare. That is a bit of an injury you must play through... Low back issues are rarely something that keeps our players out - max one to two days" (HC7; L42-44).

Chiropractors are not perceived as professionally unique and some health care co-ordinators share an affinity for other service providers groups that are perceived to provide a service equivalent to chiropractors. In instance of financial limitations co-ordinators may look towards a core provider team that may also be able to provide some of manual therapy options associated with chiropractic. The relevance of a professional group with a niche interest in the spine is questioned in the context of football.

\section{Barriers and facilitators}

Based on respondent feedback, we identified 4 barriers and 3 facilitators relevant to chiropractor involvement in this context of practice. These are summarized in table 1. (see also table 1).

\section{Table 1: Barriers and facilitators to chiropractor inclusion in inter-professional practice.}

When not included as service providers, we observed two barriers relating to the expertise chiropractors are perceived to offer. Firstly, and in a general sense, back pain was perceived as self-remitting. And as a consequence, the need for a back pain expert was questioned. Secondly, the uniqueness of chiropractors as providers of manual therapy interventions was queried, with occupational groups. Specifically, osteopaths were perceived to have similar utility and were suggested as an alternative.

Professional groups, in this instance physiotherapists, appear to compete with chiropractors for positions as in-house service providers.

Budgetary limitations, again in a general sense appear reduce the level of chiropractor service utilisation. However, perhaps more importantly, it would appear that with a tight health care budget, the co-ordinator is likely to create a lean health care team, composed of providers with known roles.

With regards to facilitators, health care co-ordinators incorporating chiropractic services, endorsed the notion of the interprofessional team, offering a superior health care solution, due to a larger pool of shared knowledge. Moreover, and with a focus on back and neck-related problems, chiropractors were specifically seen as a provider group with an important contribution to offer. Lastly, the utility of chiropractors as part of the in-house provider team, was strongly endorsed by athlete responders. This 
perspective appears grounded in the need for regular contact with chiropractors to manage long-term health care issues.

\section{Discussion}

\section{Role}

Health care co-ordinators expressly engaged in-house chiropractors as musculoskeletal health experts offering discipline-specific knowledge and with a role in clarifying biomechanical injury mechanisms, undertaking patient assessment and diagnosis and providing a variety of conservative manual interventions. In this regard, our results differ from previously reported data, and in particularly those of Therberge (11), who reported the engagement of chiropractors in a limited role and based on pressure created by athletes.

However, this role designation stood in contrast with clubs not engaging the services of a chiropractor. In these instances, osteopaths were instead utilized in a specific therapist role providing a particular manual intervention and were excluded from participation in rehabilitation protocols. Perhaps somewhat unsurprising, chiropractors were not perceived as having a unique professional role. This finding is more in-tune with previous studies describing the designated role of chiropractors functioning as a part of health care teams $(11-13)$.

\section{Value}

The perceived value chiropractors as part of the in-house health care team, lay in the broadening of the shared pool of knowledge, yielding better diagnoses and more comprehensive management strategies. Theoretically speaking, this perceived value relates to the notion of cognitive maps, where a multidisciplinary cognitive map offered by the IPHCT, is more efficient than a mono-disciplinary one (the single practitioner), for developing accurate, strong problem-solving routines (32).

A novel observation and operationalization of added value, emerged from instances, where chiropractors transitioned from from external to in-house practitioners. In particular, with direct access to athletes, the chiropractor was able to timeously initiate appropriate care, and in this way better maintain individualized care strategies. This improved care continuity is in-tune with the athlete-centered focus to care considered desirable in this practice context $(6,33)$.

\section{Salient issues}

A key perceived driver for the development of interprofessional teams is strategic organisational policy (32). In this regard, our data is suggestive of the increased involvement of chiropractors within more affluent clubs. However, IPP is also thought to thrive in collaboration-rich settings $(3,4)$. From the responder feedback, the decision to engage in-house chiropractors was based on the evolution of successful collaborative practice or a combination of these factors. Our data did not elucidate the issue of strategic health care policy. 
Our data suggests, that chiropractors have claimed a role as musculoskeletal health expert in Superliga clubs. However, it was not possible to discern, whether this role was generalised or limited to spinal complaints. It stands to reason that with a generalized role, the chiropractor is more likely to achieve core member status, rather than remaining a value-added service provider.

\section{Strengths and limitations}

Our sampling process allowed for the observation of contrasting cases; the third sampling category, that of the externally contracted chiropractor was particularly useful in clarifying the developing practice role of chiropractors.

The role of osteopaths as service providers was only observed from the perspective of health care coordinators. A thicker description and triangulation with osteopaths would have provided greater saturation.

\section{Conclusion}

When engaged as in-house team members, chiropractors are engaged in IPP characterized by meaningful collaboration. Their presence is perceived to improve the quality of athletic health care through the addition of discipline-specific knowledge, diagnostic triage capabilities and increased intervention options. In instances where chiropractors are not utilized, the role of the spinal health expert appears to be limited to that of a technician/therapist. It is unclear whether chiropractors can claim core team member status. Further exploration of this interesting context of IPP practice is warranted.

\section{Declarations}

Ethics approval and consent to participate

The study followed the guidelines of the Danish Data Protection Agency and was conducted with the approval of the science and ethics committee of the Danish region of Southern Denmark (Reference number: 20202000-85).

Consent for publication

Not applicable

Availability of data and materials

Interview transcripts are available on request. 
Competing interests

The authors declare no competing interests in relation to this work

\section{Funding}

This project was not a funded project.

Authors' contributions

$\mathrm{JH}-$ Methods, Data collection, analysis, first article draft, article revision

AK-Methods, Data collection, analysis, article revision

CM- Conceptualisation, design of the work, interpretation of data, article revision

Acknowledgements

Not applicable

\section{References}

1. Parse RR. Interdisciplinary and interprofessional: what are the differences? Los Angeles: Sage Publications Sage CA; 2015.

2. WHO. Framework for action on interprofessional education and collaborative practice. Geneva: World Health Organization; 2010.

3. Buring SM, Bhushan A, Broeseker A, Conway S, Duncan-Hewitt W, Hansen L, et al. Interprofessional Education: Definitions, Student Competencies, and Guidelines for Implementation. Am J Pharm Educ. 2009;73(4):59.

4. Franklin CM, Bernhardt JM, Lopez RP, Long-Middleton ER, Davis S. Interprofessional teamwork and collaboration between community health workers and healthcare teams: An integrative review. Health services research managerial epidemiology. 2015;2:2333392815573312.

5. CAIPE: The Centre for the Advancement of Interprofessional Education; 2019 [Available from: https://www.caipe.org/.

6. Holm S, McNamee M. Ethics in sports medicine. BMJ. 2009;339:b3898.

7. Myburgh C, Hansen T, Holm Beck A, Boyle E. Cracking the code for maintaining quality training in Olympic distance triathlon: lessons learnt from a squad of elite Scandinavian athletes. BMJ open 
sport exercise medicine. 2017;3(1):e000274.

8. Breitbach AP, Richardson R, Education NATAECf, Education I, Group PiATW. Interprofessional Education and Practice in Athletic Training. Athletic Training Education Journal. 2015;10(2):170-82.

9. Julian C, Hoskins W, Vitiello AL. Sports chiropractic management at the World Ice Hockey Championships. Chiropr Osteopat. 2010;18:32-.

10. Bigouette JP, Owen EC, Greenleaf J, James SL, Strasser NL. Injury Surveillance and Evaluation of Medical Services Utilized During the 2016 Track and Field Olympic Trials. Orthopaedic journal of sports medicine. 2018;6(12):2325967118816300.

11. Theberge $N$. The integration of chiropractors into healthcare teams: a case study from sport medicine. Sociol Health IIIn. 2008;30(1):19-34.

12. Mizrachi N, Shuval JT, Gross S. Boundary at work: alternative medicine in biomedical settings. Sociol Health IIIn. 2005;27(1):20-43.

13. Shuval JT, Averbuch E. Complementary and alternative health care in Israel. Israel Journal of Health Policy Research. 2012;1(1):7.

14. Myburgh C. A QUALITATIVE EXPLORATION OF KEY INFORMANT PERSPECTIVES REGARDING THE NATURE AND IMPACT OF CONTEMPORARY, LEGISLATION ON PROFESSIONAL DEVELOPMENT. A GROUNDED THEORY STUDY OF CHIROPRACTIC IN DENMARK. J Manipulative Physiol Ther. 2014;37(6):383-95.

15. Tofft-Jørgensen L, Gottlieb P. Medlemstal. 2019: Danmarks Idrætsforbund; 2020 [Available from: https://www.dif.dk/da/politik/vi-er/medlemstal.

16. Transfer Markt

Superliga. Transfer Markt; 2020 [Available from: ttps://www.transfermarkt.com/superligaen/marktwerteverein/wettbewerb/DK1.

17. Winther Sørensen J. Medical Staff Audit- 2018. Copenhagen: F.C. København; 2018.

18. Hsieh C-e. Strengths and weaknesses of qualitative case study research. University of Leicester Publishing. 2004.

19. Adom D, Yeboah A, Ankrah A. Constructivism philosophical paradigm: implication for research, teaching and learning. GJAHSS. 2016;4:1-9.

20. Babbie EaM. J. The practice of social research. Cape Town: Oxford University Press South Africa; 2001.

21. Baxter P, Jack S. Qualitative case study methodology: Study design and implementation for novice researchers. The qualitative report. 2008;13(4):544-59.

22. Coyne IT. Sampling in qualitative research. Purposeful and theoretical sampling; merging or clear boundaries? Journal of advanced nursing. 1997;26(3):623-30.

23. Kristensen GK, Ravn MN. The voices heard and the voices silenced: recruitment processes in qualitative interview studies. Qualitative Research. 2015;15(6):722-37.

24. Ness LR. Are we there yet? Data saturation in qualitative research. 2015. 
25. Reeves S, Lewin S, Zwarenstein M. Using qualitative interviews within medical education research: why we must raise the'quality bar'. Medical Education-Oxford. 2006;40(4):291-2.

26. Lo lacono V, Symonds P, Brown DHK. Skype as a Tool for Qualitative Research Interviews. Sociological Research Online. 2016;21(2):1-15.

27. Archibald MM, Ambagtsheer RC, Casey MG, Lawless M. Using Zoom Videoconferencing for Qualitative Data Collection: Perceptions and Experiences of Researchers and Participants. International Journal of Qualitative Methods. 2019;18:1609406919874596.

28. Dicicco-Bloom B, Crabtree BF. The qualitative research interview. Med Educ. 2006;40(4):314-21.

29. Srivastava A, Thomson SB. Framework analysis: a qualitative methodology for applied policy research. 2009.

30. Polit DFTB. C. Nursing Research: Principles and Methods2004.

31. Birt L, Scott S, Cavers D, Campbell C, Walter F. Member checking: a tool to enhance trustworthiness or merely a nod to validation? Qual Health Res. 2016;26(13):1802-11.

32. Reid C, Stewart E, Thorne G. Multidisciplinary sport science teams in elite sport: Comprehensive servicing or conflict and confusion? Sport Psychol. 2004;18(2):204-17.

33. Gulbin JP, Croser MJ, Morley EJ, Weissensteiner JR. An integrated framework for the optimisation of sport and athlete development: a practitioner approach. Journal of sports sciences. 2013;31(12):1319-31.

\section{Table}

Table 1: Barriers and facilitators to chiropractor inclusion in inter-professional practice. 


\begin{tabular}{llll}
\hline rier & Key quote(s) & Facilitator & Key quote(s) \\
\hline ain & ... we do not experience players who have an issue with their & Shift toward & I found that he would be able to \\
& low back being out of training. (HC7; L42-44). & $\begin{array}{l}\text { complement me and the other } \\
\text { physiotherapists. Particularly }\end{array}$ \\
ce? & & health care & with the knowledge of the back \\
& & delivery & and neck (HC1; L52-54).
\end{tabular}

We do not [decide] from our own subject knowledge. ... we combine our subject knowledge and get a common output - and that is the output we release (HC3; L57-60).

\section{ness}

... we have had osteopaths... instead of chiropractors... which actor you can say on some things are down the same road (HC6; se $\quad$ L6-10).

I do not know the chiropractor's role in rehabilitation and how good they are at that - and that is probably why it is physiotherapists we employ (HC7; L54-58).

$g \quad$... the previous physios were not fans of chiropractors, it was ir the players who needed to ask to be seen by a chiropractor... tition (CH1; L41-44).

\section{Perceived}

need for a

(spinal) MSK

expert

Athlete

satisfaction
I think there was a need for their [chiropractor] knowledge and what they could do (HC5; L122).
We benefit much from them... The chiropractors are more long-term solution oriented. I think that is the way so many utilize them... (AT2; L20-26)

al ons

It is not that I do not want to be there more, but I do not think the club can afford it (CH1; L99-101).

There is [only] money for two full time employees and I prefer that instead of me being full time and four others sharing the other days, to be as few as possible. And from those criteria I think it makes more sense to have two full time physiotherapists... (HC7; L14-19).

\section{Figures}


Figure 1: Flowchart of the sampling process

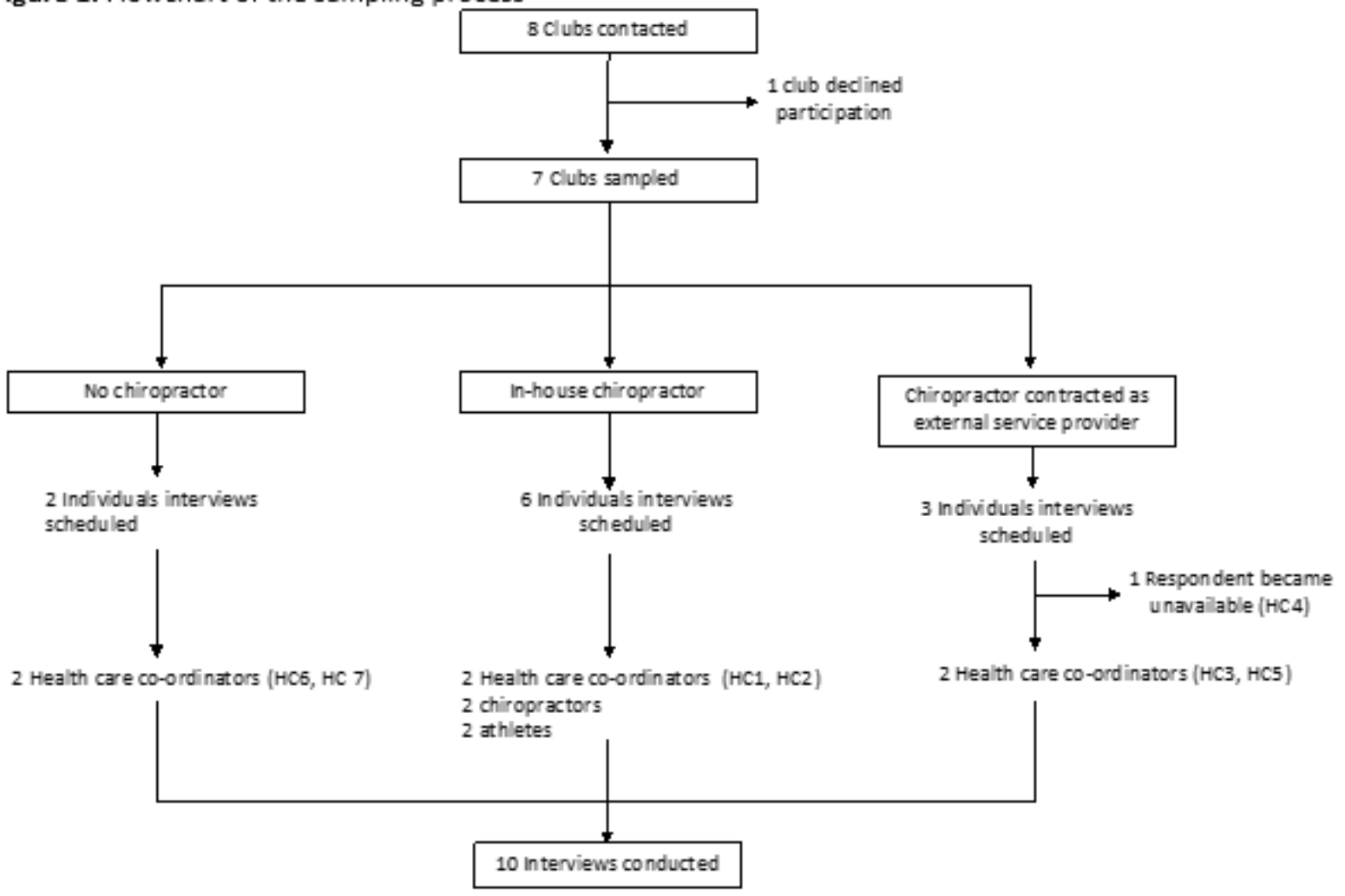

Figure 1

Flowchart of the sampling process 


\section{Codes}

Code families

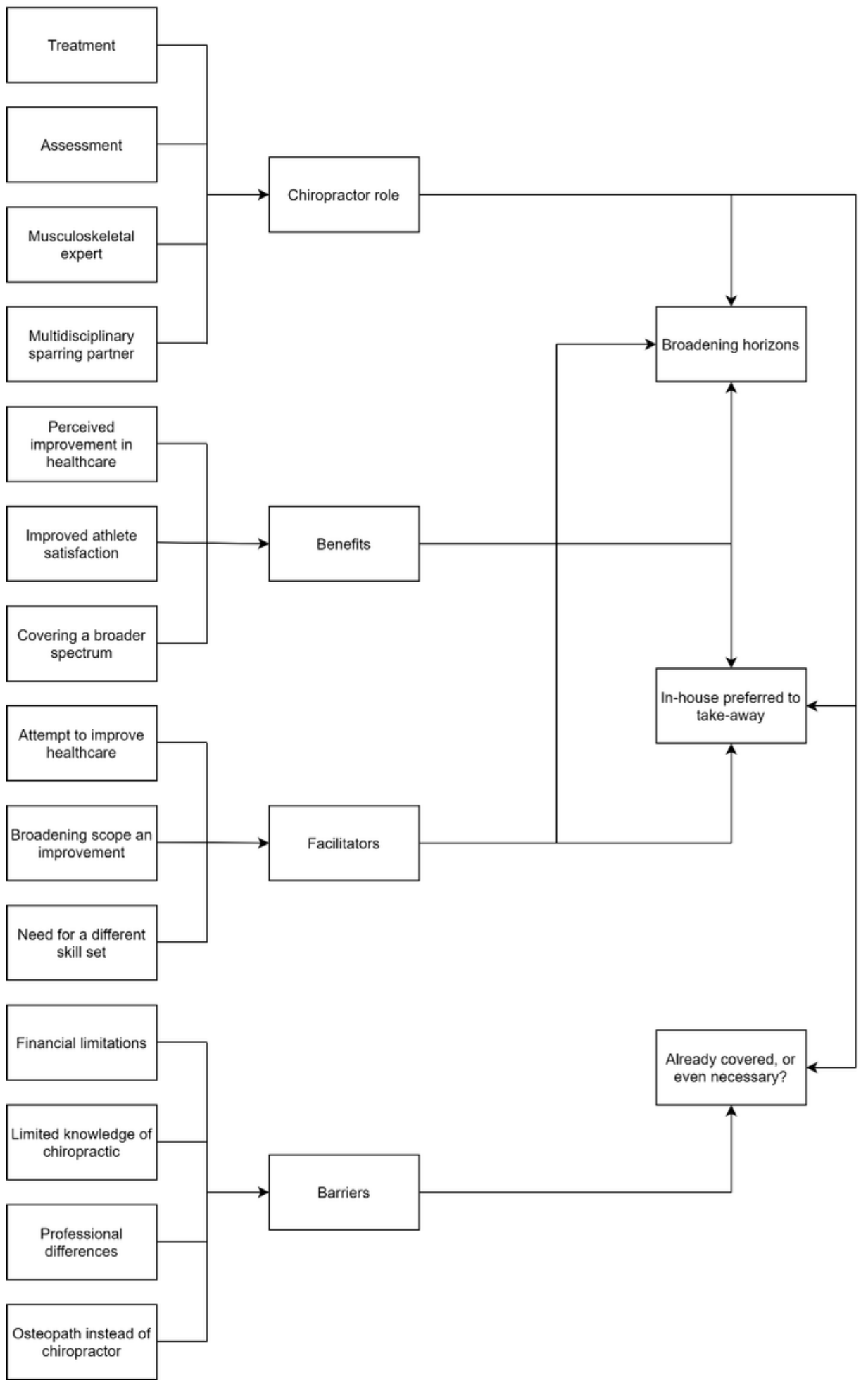

\section{Figure 2}

Organisation of data indicating emergence of themes. 